\title{
EVALUACIÓN DE LA DIAGÉNESIS ÓSEA MEDIANTE EL USO DE MICROSCOPÍA ELECTRÓNICA DE BARRIDO (MEB): APROXIMACIONES ANALÍTICAS APLICABLES A MUESTRAS ARQUEOLÓGICAS
}

\author{
Gustavo Barrientos ${ }^{1,2^{*}}$, Patricia L. Sarmiento ${ }^{3}$ y Paula E. Galligani $i^{1,2,4,5}$ \\ ${ }^{1}$ División Antropología. Facultad de Ciencias Naturales y Museo. Universidad Nacional de La Plata. La Plata. Argentina \\ ${ }^{2}$ Consejo Nacional de Investigaciones Científicas y Técnicas (CONICET) \\ ${ }^{3}$ Servicio de Microscopía Electrónica de Barrido. Facultad de Ciencias Naturales y Museo. Universidad Nacional de La Plata. La Plata. \\ Argentina \\ ${ }^{4}$ Grupo de Investigaciones Arqueológicas del Nordeste (GIAN). Rosario. Argentina \\ ${ }^{5}$ Fundación Arqueológica y Antropológica del Litoral (FUNDARQ). Santa Fe. Argentina
}

PALABRAS CLAVE hueso; alteración histológica; imágenes

\begin{abstract}
RESUMEN El objetivo del presente trabajo es realizar una revisión de las aproximaciones analíticas aplicables al estudio de la diagénesis ósea -dentro del campo más amplio de la tafonomía- que se basan en el uso de la microscopía electrónica de barrido (MEB). Para ello, se presenta a) una síntesis de los conocimientos actuales acerca de los procesos diagenéticos que afectan a la integridad histológica de los restos óseos, tanto humanos como faunísticos, en depósitos sedimentarios terrestres; b) una exposición abreviada de los principios generales de la microscopía electrónica, particularmente de la MEB y c) un comparación de las diferencias -en cuanto a requerimientos, información obtenible y venta-
\end{abstract}

jas/desventajas asociadas- entre los análisis centrados en los dos principales tipos de imágenes producidas por un MEB, es decir, las de electrones retrodispersados y las de electrones secundarios. Se concluye que, dada la disponibilidad relativamente amplia de esta tecnología, el factor crítico para una incorporación más activa del MEB en la investigación arqueológica y bioantropológica no es el acceso al equipamiento, sino la apropiada identificación de los problemas de investigación a resolver, así como la adecuada selección y ejecución de las técnicas de observación y análisis a emplear. Rev Arg Antrop Biol 18(2), 2016. doi:10.17139/ raab.2016.0018.02.03

\section{KEYWORDS bone; histological alteration; images}

ABSTRACT The aim of this paper is to review analytic approaches to bone diagenesis based on the use of scanning electron microscopy (SEM). In order to do this, we present a) a synthesis of current knowledge about diagenetic processes affecting the histological integrity of human and faunal skeletal remains in terrestrial sedimentary deposits; b) a brief description of the general principles of electron microscopy, particularly of SEM, and c) a comparison of the main differences -in terms of requirements, recoverable information, and associated pros and cons- between analyses centered on the use of either of the two main kinds of images produced by a SEM, i.e., backscattered electron images and secondary electron images. It is concluded that, given the relatively wide availability of this technology, the critical factor to a more active involvement of SEM in archaeological and bioanthropological research is not the access to equipment, but the correct identification of the research problems to be solved, as well as the proper selection and implementation of the observational and analytical techniques to be employed. Rev Arg Antrop Biol 18(2), 2016. doi:10.17139/raab.2016.0018.02.03
El término tafonomía fue propuesto inicialmente por el paleontólogo ruso Ivan A. Efremov para definir tanto un campo de estudio como un método, vinculado con la paleontología y la geología y orientado a proveer conocimiento acerca del paso de los restos orgánicos desde la biósfera a la litósfera (Efremov, 1940: 85). En el campo de la arqueología, el interés por la tafonomía fue creciendo a partir de la década de 1970, en el contexto del estudio de los procesos de formación del registro y de la obtención de datos paleobiológicos, paleoclimatológicos y paleoecológicos (Behrensmeyer y Hill, 1980;
Gifford, 1981; Nash y Petraglia, 1987), adoptándose primero como una herramienta esencial en los trabajos zooarqueológicos (ver revisiones en Lyman, 1994, 2010) y más tarde, en los estudios bioarqueológicos y forenses (v.g. Haglund

Financiamiento: Universidad Nacional de La Plata (N740).

*Correspondencia a: Dr. Gustavo Barrientos. División Antropología. Facultad de Ciencias Naturales y Museo. Universidad Nacional de La Plata. La Plata 1900. Buenos Aires. Argentina. E-mail: barrient@museo.fcnym.unlp.edu.ar

Recibido 2 Diciembre 2014; aceptado 30 Abril 2015

doi:10.17139/raab.2016.0018.02.03 
y Sorg, 1997; White y Folkens, 2005; Duday, 2009; Ortiz López, 2011; Pokines y Symes, 2013). En el contexto de los estudios tafonómicos -entendidos éstos como constitutivos de un campo orientado al conocimiento de la historia pre y postdepositacional de la totalidad de los componentes materiales del registro arqueológico (i.e. tafonomía irrestricta, en el sentido de Borrero, 2011: 269)-, la diagénesis se refiere a los procesos de alteración o modificación que experimentan tales materiales con posterioridad a su enterramiento ( $c f$. Lyman, 1994: 417). En el caso de los tejidos de los vertebrados, particularmente de los tejidos duros (i.e. huesos y dientes), la diagénesis involucra un conjunto de cambios físicos y/o químicos ocurridos a diferentes profundidades (i.e. alteraciones superficiales e internas) y niveles estructurales (i.e. alteraciones macro-, micro- y ultraestructurales) de los elementos afectados. Las modificaciones diagenéticas incluyen, entre otras, la descomposición, erosión, cementación, mineralización, disolución y deformación de los tejidos, como consecuencia de la interacción entre factores intrínsecos (e.g. tamaño, porosidad, estructura química y molecular de huesos y dientes) y extrínsecos (química y dinámica de los suelos, acción de microorganismos y regímenes hidrológicos y de temperatura a los que están sometidos los depósitos sedimentarios) (Von Endt y Ortner, 1984; Lyman, 1994; Tütken y Vennemann, 2011; Hollund et al., 2014).

En el caso de los restos óseos, tanto humanos como faunísticos, el estudio de los efectos diagenéticos incluye una variedad de parámetros tales como el grado de integridad histológica (Hedges et al., 1995), contenido de proteínas (Collins y Galley, 1999), relación carbono/ nitrógeno (Ambrose, 1990), incremento de la porosidad (Hedges et al., 1995), aumento de la cristalinidad (Weiner y Bar-Yosef, 1990) y el incremento de la cantidad de microfisuras (Jans et al., 2002). La variación en los valores de dichos parámetros, así como el grado de correlación existente entre ellos, depende del grado de descomposición y alteración de los componentes orgánicos e inorgánicos del hueso, que resultan de procesos vinculados con la actividad de microorganismos, la composición de los suelos y los regímenes hidrológicos imperantes en los respectivos ambientes de depositación (Hedges y Millard, 1995; Hedges et al., 1995; NielsenMarsh y Hedges, 2000; Collins et al., 2002; Jans et al., 2004; Smith et al., 2007; Turner-Walker, 2008). En el caso específico de la integridad histológica, la causa principal de alteración es la actividad de hongos y bacterias en ambientes terrestres y de cianobacterias, ameboides $\mathrm{y}$ algas en ambientes marinos (Marchiafava et al., 1974; Hackett, 1981; Ascenzi y Silverstrini, 1984; Piepenbrink, 1986; Child, 1995; Jans et al., 2004; Bell y Elkerton, 2008; Turner-Walker, 2008). Por este motivo, el grado de alteración histológica es el principal indicador de una de las cuatro trayectorias diagenéticas identificadas para los huesos humanos y faunísticos, a saber, la denominada "ataque microbiano" (sensu Smith et al., 2007).

La evaluación del grado de preservación de las estructuras histológicas en muestras óseas de origen arqueológico se ha realizado, hasta el presente, mediante una variedad de técnicas de observación, que incluyen microscopía óptica (MO) (e.g. Stout, 1978; Hackett, 1981; Hanson y Buikstra, 1987; Hedges et al., 1995; Gutiérrez, 2001; Jans et al., 2002; FernándezJalvo et al., 2010; Hollund et al., 2012a), microscopía electrónica de transmisión (MET) (e.g. Hackett, 1981; Ascenzi y Silverstrini, 1984; Pesquero et al., 2010) y microscopía electrónica de barrido (MEB) (e.g. Hackett, 1981; Bell 1990; Barrientos 1997; Jackes et al., 2001; Turner-Walker y Syversen, 2002; Turner-Walker y Jans, 2008; Fernández-Jalvo et al., 2010; Pesquero et al., 2010; Pesquero y Fernández-Jalvo, 2014). Debido a la multiplicación de estudios microscópicos orientados a la evaluación del grado de diagénesis ósea, particularmente mediante el uso de MEB, resulta necesario discutir las ventajas y desventajas de las diferentes técnicas existentes en relación con una serie de variables tales como tiempo, recursos, facilidades disponibles y cantidad/calidad de la información recuperada. En este contexto, el objetivo del presente trabajo es realizar tal discusión, para lo cual se presentará previamente: a) una síntesis de los conocimientos actuales acerca de los procesos diagenéticos que afectan a la integridad histológica de los restos óseos en depósitos sedimentarios terrestres, así como de las vías para caracterizar tal integridad, b) una exposición abreviada de los principios generales de microscopía electrónica, 
particularmente de MEB y c) un compendio de las diferencias -en cuanto a requerimientos, ventajas y desventajas asociadas- entre los análisis centrados en las dos principales fuentes de información producidas por MEB, i.e. las imágenes de electrones retrodispersados y las imágenes de electrones secundarios.

\section{Diagénesis ósea}

\section{La histología normal del hueso y sus alte- raciones (bioerosión)}

El hueso es un tejido compuesto, con una estructura jerárquica multiescalar (Rogel et al., 2008; Turner-Walker, 2008; Weiner, 2010) (Fig. 1). Este tejido está constituido por una fase mineral, conformada por pequeños cristales de bioapatita (i.e. un fosfato de calcio cristalino análogo a la hidroxiapatita), que se encuentra estrechamente vinculada, en el nivel estructural más profundo -i.e. en el nivel sub-nanoestructural (Rogel et al., 2008)-, con una fase orgánica conformada con una matriz de proteína, predominantemente colágeno de Tipo I (Turner-Walker, 2008; Tütken y Vennemann, 2011). Las moléculas en forma de triple hélice de colágeno, junto con los cristales de bioapatita que se disponen entre las mismas, se asocian entre sí en forma paralela y escalonada constituyendo, sucesivamente, fibrillas y fibras que componen el soporte estructural de los huesos (nivel nanoestructural; Rogel et al., 2008). En el siguiente nivel (i.e. sub-microestructural; Rogel et al., 2008), las fibras se disponen en capas cilíndricas superpuestas denominadas lamelas, en el centro de las cuales existe un canal, denominado de Havers que, en el hueso vivo, da paso a pequeños vasos sanguíneos y fibras nerviosas. En la zona de unión de las lamelas concéntricas se encuentran micro-cavidades denominadas lagunas, cada una de ellas habitada por una célula ósea u osteocito. Los osteocitos emiten prolongaciones citoplasmáticas que atraviesan las lamelas a través de una red de microcanales llamados canalículos, los cuales comunican a las lagunas entre sí y a éstas con el canal de Havers. Un segundo tipo de canales, llamados de Volkmann, corren perpendicularmente al eje longitudinal del hueso, actuando como conectores entre los canales de Havers contiguos (Ross y Pawlina, 2007).

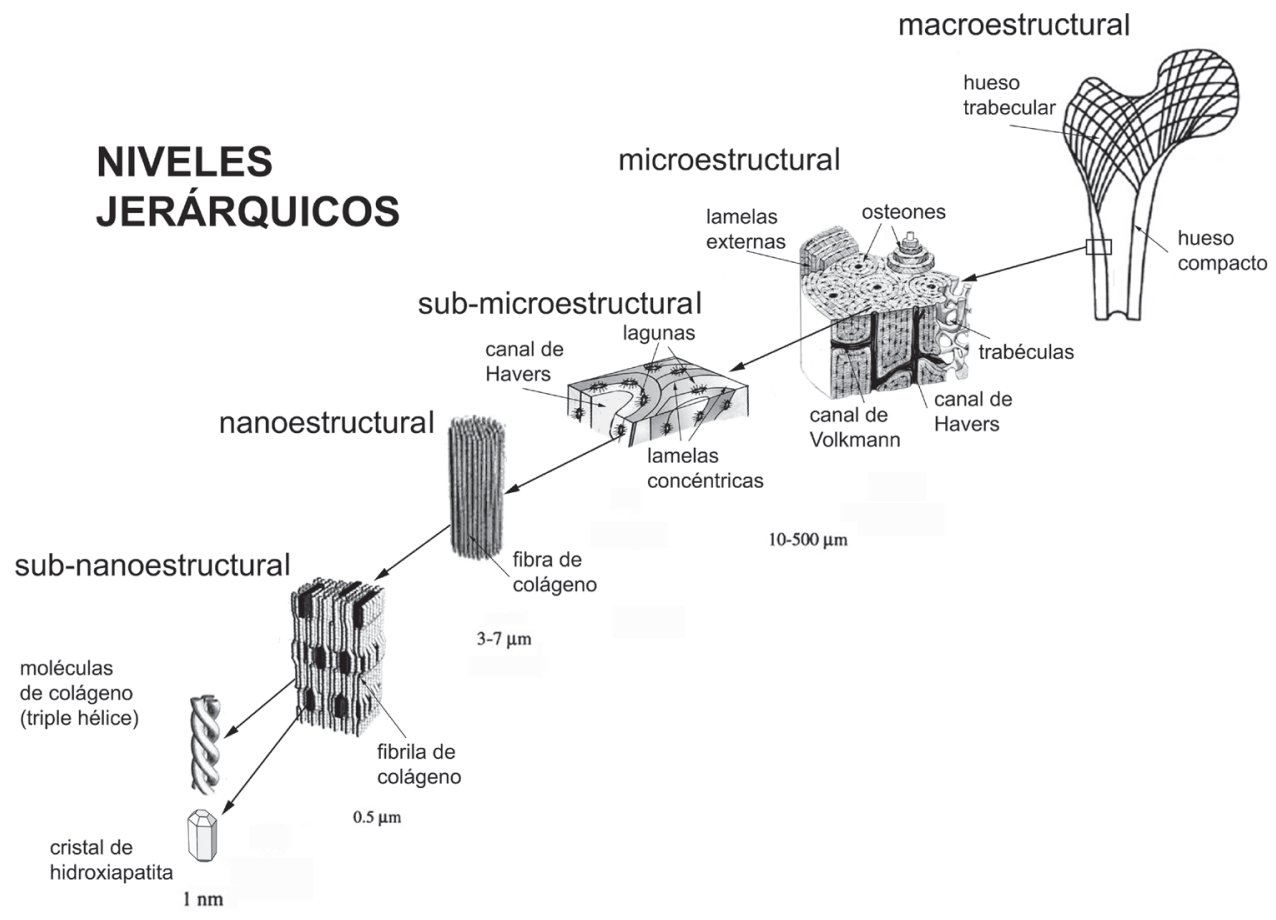

Fig. 1. Diferentes niveles jerárquicos de la estructura ósea (modificado a partir de Rogel et al., 2008). 
El conjunto conformado por las lamelas concéntricas, las lagunas, los canalículos y el canal de Havers, recibe el nombre de osteón o sistema de Havers constituyendo, en el nivel microestructural, la unidad fundamental del hueso (principalmente del hueso compacto o lamelar) (Weiner, 2010). Entre los osteones contiguos existen lamelas incompletas, denominadas intersticiales, mientras que, rodeando al hueso compacto tanto en su parte externa como interna, existen lamelas llamadas circunferenciales (Ross y Pawlina, 2007; Weiner, 2010).

Los procesos diagenéticos que afectan a las fases mineral y orgánica de los huesos se manifiestan a distintos niveles estructurales, abarcando una amplia gama de agentes y condiciones (Von Endt y Ortner, 1984; Lyman, 1994; Hedges y Millard, 1995; Hedges et al., 1995; Collis et al., 2002; Turner-Walker, 2008; Tütken y Vennemann, 2011). En el caso de la pérdida de la integridad histológica -que se resuelve, principalmente, en el nivel microestructural-, el principal factor causal es la actividad microbiana que, en ambientes terrestres, incluye a hongos y bacterias (Jans et al., 2004) (para un resumen del estado actual del conocimiento acerca del ataque biológico a los huesos en ambientes marinos, ver Bell y Elkerton, 2008). Por esta razón, el conjunto de los cambios físicos ocurridos a nivel histológico en los huesos se encuadran dentro de los procesos de bioerosión (Nielsen-Marsh y Hedges, 2000; Jans, 2008; Turner-Walker y Jans, 2008; TurnerWalker, 2012; Hollund et al., 2014). El conocimiento de tales procesos es importante por cuanto se ha demostrado que la bioerosión: a) causa la pérdida de colágeno del hueso (Hedges 2002); b) promueve la introducción de $\mathrm{ADN}$ bacteriano y/o fúngico (Hollund et al., 2014); c) aumenta la porosidad, lo que conduce a una descomposición acelerada del tejido y a una mayor contaminación (Gilbert et al., 2005) -principalmente por sustancias tales como ácidos húmicos y compuestos de hierro y cobre-, que pueden causar problemas tanto para la extracción del colágeno como del ADN (Breen y Murphy, 1995; Van Klinken y Hedges, 1995; King et al., 2009; Alaeddini et al., 2010). Por estos motivos, el análisis de la histología del hueso mediante el uso de diferentes técnicas microscópicas resulta un medio adecuado para conocer el estado de las muestras antes de la realización de estudios específicos (e.g. isotópi- cos, paleogenéticos) (Hollund et al., 2014) y para inferir trayectorias diagenéticas (Hedges, 2002; Smith et al., 2007; Turner-Walker y Jans, 2008; Hollund et al., 2012b).

\section{Causas de la alteración histológica del hueso}

Se ha comprobado que, en restos óseos humanos arqueológicos procedentes de entierros intencionales, predomina el ataque bacteriano sobre el fúngico mientras que, en los restos faunísticos, ocurre la situación inversa (Jans et al., 2004). La explicación de esta observación reside en el diferente estado en el que ingresan los huesos humanos y faunísticos a los depósitos sedimentarios: mientras que los primeros lo hacen habitualmente formando parte cadáveres completos (i.e. entierros primarios), los segundos -típicamente- lo hacen desarticulados y desprovistos de otros tejidos. En un cadáver, el ataque a los huesos puede ocurrir a partir de bacterias endógenas como de bacterias presentes en los suelos, sobre todo en depósitos sedimentarios utilizados recurrentemente como áreas de entierro. Tales suelos pueden albergar poblaciones latentes de bacterias capaces de utilizar el colágeno óseo como substrato para su crecimiento (Jans et al., 2004; Turner-Walker, 2008). La mayor parte de los huesos de animales que ingresan a los depósitos como consecuencia de actividades de consumo y descarte humano, no pueden ser afectados por bacterias endógenas; sin embargo, su relativamente buena preservación inicial los hace atractivos como fuentes de nutrientes para los hongos presentes en el suelo (e.g. géneros Mucor y Fusarium; Hackett, 1981), que requieren condiciones apropiadas para su proliferación tales como presencia de oxígeno y cierto nivel de humedad ( 20\%) (Jans et al., 2004).

A partir del ataque microbiano, la mayor parte del colágeno se pierde, incrementándose la porosidad del hueso y decreciendo así su resistencia. En efecto, los microorganismos alteran la integridad histológica del hueso a través de la destrucción focal microscópica (DFM; sensu Hacket, 1981), consistente en la construcción de túneles o galerías a través del tejido compacto del hueso (Stout, 1978; Hackett, 1981; Hanson y Buikstra, 1987; Garland, 1989; Bell, 1990; Child, 1995; Hedges et al., 1995; Jans et al., 2002, 2004; Turner-Walker y Jans, 2008). En la mayoría de los casos, los poros naturales del 
hueso (e.g. canales de Havers) parecen ser las vías preferenciales de ingreso de los microorganismos invasores, particularmente de bacterias (Jans et al., 2004). En un hueso largo, el daño microbiano se inicia típicamente en las superficies corticales externa e interna más o menos al mismo tiempo, extendiéndose luego hacia el centro del tejido compacto (Hackett, 1981).

Las bacterias degradan el colágeno a partir de la producción de enzimas colagenolíticas. Sin embargo, como en el nivel sub-nanoestructural las fibrillas de colágeno están íntimamente asociadas con los cristales de bioapatita, la efectiva actividad lítica de las enzimas sobre la proteína depende de la disolución previa de la fase inorgánica del hueso, lo cual se logra mediante la acción de ácidos orgánicos extracelulares. Debido a ello, tanto la solubilización y redepositación del mineral como el incremento de la porosidad del hueso, son los indicadores a nivel microestructural de la acción microbiana y por lo tanto, los rasgos principales a tener en cuenta en una evaluación histológica del grado de diagénesis o bioerosión ósea (Turner-Walker y Syversen, 2002).

\section{Evaluación del grado de alteración his- tológica}

Como fuera mencionado, los criterios para evaluar el grado de alteración histológica de una muestra mediante el uso de microscopía óptica u electrónica, se basan en la detección de un conjunto de cambios en la morfología normal del hueso, observables en los niveles micro y sub-microestructural. En un estudio pionero, Hackett (1981) describió cuatro tipos principales de DFM: a) lineal longitudinal, b) florecida (budded), c) lamelada (lamellate) y d) en túnel centrífugo o Wedl. Se considera que los tres primeros son producidos por bacterias mientras que el último sólo por hongos (Hackett, 1981; Jans et al., 2004), aunque se ha informado que las cianobacterias son también capaces de originar túneles Wedl (Turner-Walker y Jans, 2008). Hasta el presente sin embargo, se conoce poco acerca de las especies involucradas en cada tipo de bioerosión y de los procesos mediante los cuales los microorganismos alteran los tejidos óseos y dentales, resultando poco claro si las distintas categorías de DFM representan o bien la acción de diferentes especies o bien estadios diferentes de un mismo proceso (Hollund et al., 2014). En cualquier caso, las DFM estarían consistentemente reflejando las condiciones que afectaron a los huesos durante las etapas tempranas de su historia tafonómica más que a procesos a largo plazo ocurridos en sus respectivos contextos de depositación (Hedges, 2002; Smith et al., 2007).

Para cualificar y cuantificar el grado de alteración histológica de una muestra, Hedges et al. (1995) propusieron un índice denominado índice histológico $(\mathrm{IH})$ o índice histológico de Oxford (IHO), basado en una progresión de indicadores morfológicos de daño microestructural. Tal índice puede adoptar valores entre 5 (buena preservación histológica, indistinguible de un hueso moderno) a 0 (muy mala preser-
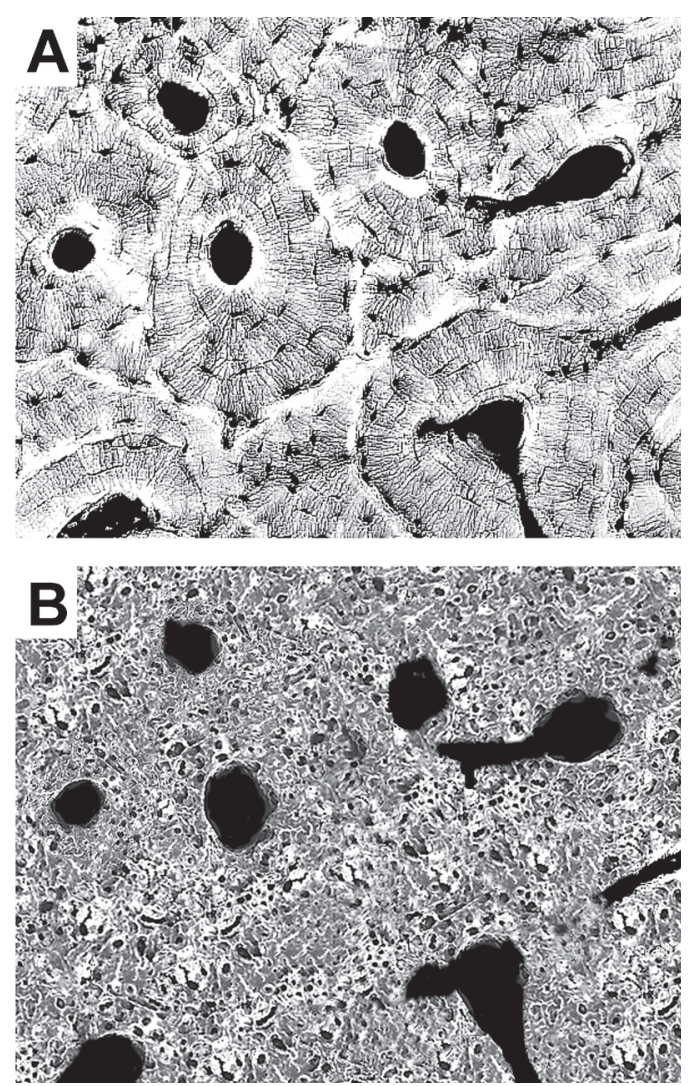

Fig. 2. Representación ideal de los extremos del Índice Histológico (IH): (A) $\mathrm{IH}=5$; B) $\mathrm{IH}=0$. Nótese la pérdida de rasgos histológicos característicos en (B) (i.e. lamelas, lagunas, canalículos; líneas de cemento), debido a la apariencia espongiforme adquirida extensivamente por el hueso; en la imagen, sólo resultan reconocibles los canales de Havers y de Volkmann. 
vación histológica, ausencia de rasgos microestructurales reconocibles) (Fig. 2). El IH no toma en cuenta el o los tipos de destrucción focal microscópica presentes en una muestra, sino la extensión y las características globales del daño observado. Desde su introducción en la literatura, ha sido aplicado en diversos estudios de diagénesis ósea, tanto mediante observaciones realizadas con microscopía óptica como electrónica (e.g. Gutiérrez, 2001; Jans et al., 2004; Shin 2011; Galligani, 2013; Morales et al., 2014). Otros autores también han utilizado escalas para caracterizar el grado de alteración histológica de muestras óseas. Por ejemplo, Prowse et al. (2004) diferencian 4 estados del hueso en relación con la presencia/ausencia, extensión y tipo de alteración: a) normal, sin evidencias de alteración de la estructura ósea, con osteones claramente visibles (equivalente al valor 5 del $\mathrm{IH}$ ); b) focalizado, con focos de degradación (aproximadamente equivalente a los valores 4, $3,2$ y 1 del $\mathrm{IH})$; c) amorfo, con degradación extendida, sin osteones visibles en el campo de observación (equivalente al valor 0 del $\mathrm{IH}$ ); d) mineralizado, con evidencias de recristalización del tejido (sin equivalente en el $\mathrm{IH}$ ).

En el estudio más comprehensivo realizado hasta el presente acerca de la correlación entre distintos parámetros diagenéticos $(\mathrm{n}=10)$, Smith et al. (2007) encontraron que el valor de $\mathrm{R}^{2}$ más alto (-0.72) corresponde al par IH-porosidad media (i.e. volumen de los poros comprendidos en el rango $>0.1 \mathrm{~mm}<8.5 \mathrm{~mm}$ de diámetro/volumen del hueso; Turner-Walker et al., 2002). Asimismo, Prowse et al. (2004) señalan el poder predictivo de la preservación histológica sobre el contenido de colágeno de los huesos, ya que las muestras identificadas como "normales" tienden a tener mayor contenido de colágeno que las categorizadas respectivamente como "focalizadas", "amorfas" y "mineralizadas" (ver asimismo, Hedges et al., 1995; Nielsen-Marsh y Hedges, 2000).

\section{Conceptos generales de microscopía electrónica}

\section{Principios físicos}

El microscopio electrónico, cuyo desarrollo inicial se produjo en la década de 1940, funciona mediante un principio similar al microscopio óptico, sólo que utiliza un flujo de electrones en lugar de un haz de luz (fotones) (Goodhew et al., 2001). En el microscopio óptico, tanto la resolución (i.e. separación máxima entre los detalles que pueden distinguirse) como el nivel de magnificación del objeto (i.e. aumento), están limitados por la longitud de onda de la luz visible. Cualquier objeto más pequeño que la mitad de la longitud de onda de la fuente de iluminación del microscopio, no puede ser observado. Debido a que la longitud de onda mínima de la luz visible (extremo violeta del espectro) es de $\sim 400 \mathrm{~nm}$, con un microscopio óptico no pueden verse objetos de tamaño menor a aproximadamente $200 \mathrm{~nm}$ (i.e. el tamaño de una bacteria mediana/pequeña). En la práctica, sin embargo, el nivel de resolución varía en función de la calidad de la lente del microscopio, por lo que rara vez se alcanza el límite de resolución arriba mencionado (Davidson y Abramowitz, 2002; Mertz, 2009; Murphy y Davidson, 2013).

Con el microscopio electrónico, en virtud de que la longitud de onda de los electrones es aproximadamente 100.000 veces menor que la de la luz visible $(0.0037 \mathrm{~nm})$, se puede alcanzar una resolución y un aumento varios órdenes de magnitud mayores que con el microscopio óptico (Goodhew et al., 2001; Mehta, 2012). El haz de electrones generado por una fuente (e.g. filamento de tungsteno, filamento de hexaboruro de lantano, emisor de efecto de campo), se enfoca por lentes electromagnéticas convergentes e interactúa -a nivel atómico- con el objeto de estudio (Goodhew et al., 2001; Mehta, 2012), generando señales que son captadas por alguno de los detectores del microscopio e interpretadas en términos de imágenes, sobre las cuales pueden llevarse a cabo diversos estudios específicos (Egerton, 2005).

\section{Tipos de microscopios electrónicos}

Existen fundamentalmente dos tipos de microscopios electrónicos: el de transmisión (MET) y el de barrido (MEB) (Goodhew et al., 2001; Egerton, 2005). El primero opera de modo similar al microscopio óptico, ya que la manera en que actúa un campo electromagnético sobre un haz de electrones es análoga a la acción de una lente de cristal sobre un haz de fotones. Utiliza, para formar la imagen, electrones transmitidos, los cuales atraviesan la muestra, sufriendo 
una absorción parcial por parte de la misma. Los electrones que logran traspasarla, colisionan contra una pantalla fluorescente y permiten formar una imagen bidimensional (plana) del objeto observado. Para este tipo de microscopios, la muestra debe ser pequeña y delgada (usualmente con espesores entre $10 \mathrm{~nm}$ y $1 \mu \mathrm{m}$ ) ya que de otro modo, los electrones tendrían mayores posibilidades de dispersarse dentro de la muestra, así como también de ser absorbidos en lugar de ser transmitidos (Egerton, 2005).

Por el contrario, el MEB utiliza electrones dispersados a partir de la superficie del objeto observado. Las señales eléctricas emitidas se convierten en una imagen, que puede observarse en una pantalla de TV o en un monitor de computadora (Ávalos, 2013). Aunque no tiene la misma resolución que el MET (el cual alcanza hasta $0,5 \mathrm{~nm}$ frente a $10 \mathrm{~nm}$ que logra el MEB; Egerton, 2005), las ventajas que presenta el MEB son que las muestras pueden ser voluminosas y que las imágenes obtenidas poseen información tridimensional. En cuanto a la preparación de las muestras, la misma es relativamente más sencilla, aunque deben realizarse ciertos procedimientos de adecuación tales como limpieza, deshidratación, secado y montaje. El objeto a observar debe ser, además, conductor de corriente eléctrica, lo cual sólo se cumple en el caso de los metales. En las muestras biológicas, por tanto, la superficie debe cubrirse con una capa de oro, cromo o carbono evaporado (Egerton, 2005). En los MEB convencionales, tanto la columna de electrones como la cámara portamuestras deben estar a un vacío aproximado de $10^{-6}$ torr (alto vacío).

A partir del desarrollo de la microscopía electrónica de barrido ambiental (MEBA), a fines de la década de 1980, es posible estudiar muestras hasta con un $100 \%$ de humedad relativa y sin metalizar ya que, además de trabajar en alto vacío $\left(10^{-3}\right.$ a $10^{-7}$ torr $)$, la cámara portamuestras de estos microscopios pueden funcionar también en bajo vacío (10 $10^{-2}$ a 1 torr) y en modo ambiental (1 a 20torr) (la columna de electrones se mantiene siempre en alto vacío, entre otras razones, para evitar la dispersión del haz de electrones y para permitir una mayor duración de la fuente de emisión) (Goodhew et al., 2001). Por este motivo, se pueden observar muestras sin necesidad de una preparación previa com- pleja, evitando o minimizando así el riesgo de generar modificaciones derivadas (i.e. artefactos de la técnica) (Gómez Varga, 2004). Sin embargo, la calidad y la resolución de la imagen en bajo vacío y en modo ambiental es menor que en el modo alto vacío ya que, bajo presión variable, muchos de los electrones del haz que deberían llegar a la muestra son desviados por las moléculas del aire, lo que redunda en una menor calidad de imagen a grandes aumentos.

\section{Imágenes e información elemental ob- tenibles con el uso de MEB}

Típicamente, los MEB poseen uno o más de los siguientes detectores de las señales emitidas por la interacción del haz de electrones con los átomos de la muestra: a) de electrones retrodispersados (ERD), b) de electrones secundarios (ES) y, c) de energía dispersiva de rayos X (EDX) (Goodhew et al., 2001; Egerton, 2005).

Los ERD se producen cuando electrones del haz (i.e. electrones primarios) colisionan, frontalmente, con núcleos atómicos de la muestra, siendo los primeros repelidos y expulsados hacia el exterior del objeto observado, con una intensidad (representada por el coeficiente de dispersión ๆ) que varía proporcionalmente en relación con el número atómico $(Z)$ de la muestra. La detección de estos electrones permite obtener, principalmente, imágenes que contienen información acerca de la composición superficial de la muestra. En efecto, a partir de una superficie totalmente lisa (i.e. pulida) pueden obtenerse imágenes con diferentes tonos de grises en función de la existencia, en la muestra, de fases con distinta densidad y composición química, es decir constituidas por distintos elementos, cada uno con su correspondiente valor de Z. Las fases con mayor $\mathrm{Z}$ promedio aparecerán más brillantes que las fases con un $\mathrm{Z}$ promedio menor. Debido a que los detectores de ERD están montados directamente por encima de la muestra, la señal recibida posee escasa información topográfica (Badía e Ibáñez, 1996; Goodhew et al., 2001; Egerton, 2005).

Los ES, a su vez, se originan cuando un electrón del haz pasa muy cerca del núcleo de un átomo de la muestra, proporcionando energía suficiente para causar que uno o varios de los electrones de los orbitales interiores del átomo sean expulsados hacia el exterior de la muestra. 
Estos electrones son de muy baja energía (por debajo de $5 \mathrm{eV}$ ), por lo que deben encontrarse muy cerca de la superficie para poder ser liberados. Las imágenes generadas contienen información sobre la topografía de la muestra, ya que un cambio de inclinación de la superficie de una muestra con respecto al haz incidente -aun de pocos grados- produce una variación apreciable en el número de ES. Si la superficie de la muestra es rugosa cada zona de la misma producirá una cantidad de ES diferente, dependiendo de la inclinación de cada sector, en la imagen se apreciarán zonas más iluminadas (i.e. bordes agudos o protuberancias, que producen una gran cantidad de ES) y otras más oscuras o de sombra (i.e. zonas deprimidas, que producen menor cantidad de ES detectables). La asociación de zonas iluminadas y oscuras es lo que proporciona, en este tipo de imágenes, una sensación de relieve además de una gran profundidad de foco. Por estos motivos, los electrones secundarios son ideales para la realización de estudios morfológicos, alcanzando resoluciones del orden de $1 \mathrm{~nm}$ en los equipos más modernos (Goodhew et al., 2001; Egerton, 2005).

Finalmente, en el caso de la EDX, el detector consiste en un espectrómetro que captura los rayos $\mathrm{X}$ emitidos por la muestra, al interactuar los electrones de las capas más internas de sus átomos con los electrones emitidos por la fuente del microscopio. Esta técnica, denominada espectroscopía de energía dispersiva de rayos $\mathrm{X}$ (MEB-EEDX), permite obtener un único espectro, emitido por todos los elementos de la muestra a la vez. Esto posibilita realizar análisis cualitativos de los constituyentes mayoritarios de las áreas de interés (1mm). Sin embargo, en muestras pulidas es posible realizar análisis cuantitativos, comparando la intensidad de los rayos $\mathrm{X}$ medida en la muestra con la intensidad de los rayos $\mathrm{X}$ producidos por una muestra patrón de composición conocida (Goodhew et al., 2001; Goldstein, 2003).

\section{Estudios de diagénesis con el uso de imágenes MEB}

En esta sección se describirán los principales antecedentes, requerimientos técnicos, tipo de información obtenible y ventajas/desventajas asociadas con la utilización de dos de las prin- cipales tipos de imágenes producidas por MEB, i.e. las de electrones retrodispersados y las de electrones secundarios.

\section{Imágenes de electrones retrodispersados (MEB-ERD)}

\section{Antecedentes}

Los primeros trabajos orientados a la evaluación de cambios diagenéticos en restos óseos de origen arqueológico (huesos humanos) mediante este procedimiento datan de comienzos de la década de 1990 y fueron llevados a cabo por Bell (1990; Bell et al., 1996). Posteriormente, la técnica fue utilizada por diferentes autores sobre restos óseos humanos (e.g. Jackes et al., 2001; Turner-Walker y Syversen, 2002), restos faunísticos (e.g. Turner Walker, 2012; Fernández-Jalvo et al., 2010; Pesquero et al., 2010) y en estudios comparativos (e.g. Jans et al., 2002; Turner-Walker y Jans, 2008; Pesquero y Fernández-Jalvo, 2014).

\section{Requerimientos}

Superficies pulidas, correspondientes a cortes delgados o a secciones gruesas -transversas o longitudinales- de los huesos. Para realizar los cortes, las muestras deben ser previamente tratadas mediante limpieza (e.g. ultrasonicación en agua o etanol), deshidratación (aplicación de etanol, acetona o secado en horno) e inclusión en vacío (con polimetilmetacrilato, silicona, resina epoxy o acrílica, etc.). Con posterioridad al corte, las muestras deben ser desgastadas hasta un grosor arbitrario, pulidas con abrasivos de distinto grano hasta obtener una superficie ópticamente plana, montadas sobre un portaobjetos y recubiertas, en el caso de observaciones realizadas con alto vacío, con una capa conductiva de oro, cromo o carbono evaporado (para una exposición detallada de los procedimientos, ver Turner-Walker y Mays, 2008).

\section{Información recuperable}

Los electrones retrodispersados son altamente sensibles a la existencia de diferencias sutiles en la densidad de la muestra, de modo tal que las estructuras histológicas normales del hueso tales como las lamelas, los canales de Havers, las líneas de cemento o las líneas de inversión pueden ser fácilmente identificadas. 
En especímenes alterados diagenéticamente, las imágenes generadas con el uso de MEB-ERD permiten visualizar porosidades de distinto diámetro, la morfología de los túneles causados por bacterias y hongos, la presencia de áreas desmineralizadas e hipermineralizadas, la existencia y extensión de microfisuras y la ocurrencia de inclusiones (e.g. partículas de suelo, framboides de pirita originados por bacterias reductoras de sulfato, etc.) en el interior de canales de Havers, poros y túneles (Bell, 1990; Turner-Walker y Jans, 2008; Turner-Walker y Mays, 2008).

\section{Ventajas/Desventajas}

La principal ventaja de los estudios llevados a cabo con MEB-ERD es la capacidad de la técnica para obtener información detallada, tanto cualitativa como cuantitativa, acerca del tipo y extensión de las modificaciones causadas por microorganismos en el nivel microestructural. En particular, resulta posible medir áreas afectadas por rasgos de bioerosión tales como poros y zonas de desmineralización e hipermineralización (e.g. Turner-Walker y Syversen, 2002). Entre las desventajas se encuentra la necesidad de someter a la muestra, en forma previa a la observación, a un pretratamiento costoso en términos de tiempo, recursos y acceso a facilidades específicas (ver discusión en Turner-Walker y Mays, 2008).

\section{Imágenes de electrones secundarios (MEB-ES)}

\section{Antecedentes}

La mención de observaciones de muestras óseas con MEB-ES orientadas a discutir procesos diagenéticos es mucho menos frecuente, en la literatura, que de aquellas realizadas con MEB-ERD. Con posterioridad al estudio precursor efectuado por Hackett (1981), otros autores han utilizado esta técnica tanto sobre huesos humanos (e.g. Grupe, 1995; Barrientos, 1997; $\mathrm{Hu}$ et al., 2006; Arenas Alatorre et al., 2007; Galligani, 2013) como faunísticos (FernándezJalvo et al., 2010; Morales et al., 2014; Pesquero y Fernández-Jalvo, 2014).

\section{Requerimientos}

Superficies preferentemente no pulidas (aunque pueden estarlo), correspondientes a seccio- nes gruesas -de tamaño variable- de los huesos. Pueden observarse superficies de fracturas naturales o inducidas mediante el uso de una sierra o disco de corte. A diferencia de los estudios realizados con MEB-ERD, los protocolos para la obtención y preparación de las muestras para análisis con MEB-ES no se encuentran adecuadamente descriptos en la literatura. A partir de nuestra experiencia, un procedimiento típico consistiría en la limpieza de las muestras mediante cepillado suave en seco y húmedo con alcoholes, ultrasonicación con alcohol (e.g. éter sulfúrico), deshidratación con alcohol etílico absoluto o acetona (en ambos casos de 99,5\%) y metalizado con oro (sólo para observaciones en alto vacío). Para algunas muestras, algunos de los pasos pueden eventualmente obviarse (e.g. cepillado, ultrasonicación), aunque no es recomendable.

\section{Información recuperable}

Los electrones secundarios permiten obtener imágenes con información tridimensional o topográfica del material estudiado. En huesos frescos o no alterados diagenéticamente, pueden visualizarse los rasgos normales de la microestructura ósea (e.g. lamelas, canales de Havers, lagunas, canales de Volkmann). En huesos con evidencias de bioerosión o diagénesis, pueden observarse la distribución y la extensión de la porosidad, resultando difícil distinguir con precisión entre las distintas DFM descriptas por Hackett (1981) (Fig. 3). Sin embargo, resulta posible adjudicar a cada muestra -dentro de ciertos límites- un valor del IH de Hedges et al. (1995) (Galligani, 2013; Morales et al., 2014).

\section{Ventajas/Desventajas}

La principal ventaja reside en el bajo costo de procesamiento de las muestras, resultando una técnica particularmente útil para la obtención rápida y económica de información acerca del estado general de conservación del hueso. Entre las principales desventajas se encuentran: a) la escasa posibilidad de realizar estudios cuantitativos acerca de la extensión de las áreas afectadas por rasgos de bioerosión o diagénesis y b) la actual falta de protocolos técnicos bien descriptos, y de eficacia probada, para la preparación de las muestras en forma previa a la observación. Este es un aspecto en el cual nos 


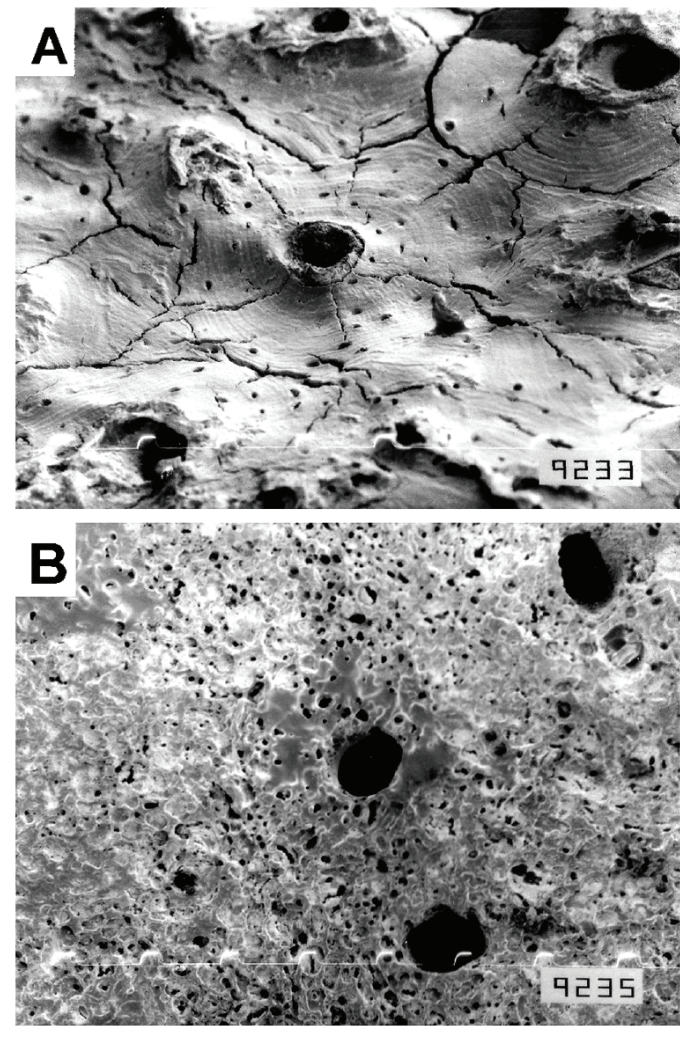

Fig. 3. Ejemplos de imágenes de electrones secundarios (MEB-ES) obtenidas a partir de huesos arqueológicos (microscopio JEOL JSM T100, Servicio de Microscopía Electrónica de Barrido, FCNyM, UNLP). En (A) (150X) se observa una buena preservación histológica, distinguiéndose claramente los canales de Havers (con probables depósitos internos de calcita), las lamelas y las lagunas; las microfisuras radiales y a lo largo de las líneas de cemento son, probablemente, un artefacto de la técnica (deshidratación y alto vacío). En (B) (150X) se observa una muy mala preservación histológica (asimilable al valor 0 del $\mathrm{IH}$ ); nótese la distribución de poros de diferente tamaño alrededor de los canales de Havers y la pérdida generalizada de rasgos microestructurales. Modificadas a partir de Barrientos (1997).

encontramos trabajando en la actualidad.

A modo de resumen, puede decirse que las diferentes técnicas de observación disponibles con el uso de MEB no son excluyentes sino complementarias, presentando cada una de ellas ventajas y desventajas que deben evaluarse en función de los objetivos y la etapa de la investigación. En este sentido, los estudios basados en el uso de imágenes de ES -en virtud de su bajo costo en términos de tiempo y recursos necesa- rios- resultan particularmente apropiados para la realización de una primera evaluación de un conjunto. A partir de tal evaluación, pueden seleccionarse aquellas muestras que requieren un estudio más detallado, tanto a través de imágenes de ERD como de espectros EDX.

\section{Consideraciones finales}

El estado de conservación de la microestructura ósea es un indicador útil desde un punto de vista tafonómico, por cuanto proporciona una idea del impacto de los procesos de bioerosión sobre los huesos (Jans et al., 2004; Jans, 2008; Turner-Walker y Jans, 2008; Hollund et al. 2012b, 2014), así como del grado de susceptibilidad de los mismos a la acción destructiva de otros agentes operantes en los depósitos sedimentarios, tales como el agua (Hedges y Millard, 1995; Hedges et al., 1995; Hedges, 2002). En este contexto, la observación de la microestructura ósea mediante el uso de MEB representa una herramienta de suma importancia, ya que proporciona -según la técnica de observación empleada- distinto tipo de información relevante no sólo para el modelado de la historia tafonómica de los conjuntos óseos analizados, sino también para la toma de decisiones, en forma complementaria con otras líneas de evidencia, acerca de los análisis que resulta viable efectuar sobre una muestra (e.g. isotópicos, moleculares, histomorfológicos, etc.) (Hollund et al., 2014). En este sentido, la técnica más económica en tiempo y otros recursos -i.e. la observación de secciones gruesas mediante el uso de imágenes de electrones secundarios- resulta particularmente útil para el filtrado inicial de muestras numerosas.

Consideramos que la incorporación más activa del MEB en los estudios arqueológicos y bioantropológicos en nuestro país puede verse favorecida por el creciente número de centros que disponen de esta tecnología, así como por la variedad de equipos y prestaciones existentes (en la actualidad, se cuenta con al menos 29 MEB en operación distribuidos en diferentes instituciones, según datos del Sistema Nacional de Microscopía del Ministerio de Ciencia y Tecnología de la Nación; http://sistemasnacionales. mincyt.gob.ar/mapa/index.html). Por consiguiente, no es tanto el acceso al equipamiento y su costo de uso -que es, dependiendo de la técni- 
ca, relativamente reducido- sino, como intentamos mostrar en este trabajo, la apropiada identificación de los problemas a resolver, así como la adecuada selección y ejecución de las técnicas de observación y análisis a emplear, los aspectos que continúan siendo críticos para el desarrollo y expansión de los estudios de microscopía electrónica dentro de nuestro campo disciplinar.

\section{LITERATURA CITADA}

Alaeddini R, Walsh SJ, Abbas A. 2010. Forensic implications of genetic analyses from degraded DNA-A review. Forensic Sci Int: Genetics Supplement Series 4:148157. doi:10.1016/j.fsigen.2009.09.007

Ambrose SJ. 1990. Preparation and characterization of bone and tooth collagen for isotopic analysis. J Archaeol Sci 17:431-451. doi:10.1016/0305-4403(90)90007-r

Arenas Alatorre JA, Sánchez Pérez S, Escalona A, Sterpone O, Zorrilla C, Gómez Serrano A. 2007. Diagénesis en huesos humanos de la época colonial del estado de Hidalgo, México. Estudios de Antropología Biológica XIII (1):361-380.

Ascenzi A, Silverstrini G. 1984. Bone-boring marine microorganism, an experimental design. J Hum Evol 13:531536. doi:10.1016/s0047-2484(84)80006-8

Ávalos M. 2013. La microscopía electrónica de barrido: una ventana al mundo micro. Revista de la Sociedad Argentina de Histotecnología 24:6-9.

Badía JM, Ibáñez J. 1996. Electrones retrodispersados y absorbidos. En: Aballe Carride M, López Ruiz J, Adeva Ramos P, Badía Pérez JM, coordinadores. Microscopía electrónica de barrido y microanálisis por rayos X. Madrid: CSIC. p 73-99.

Barrientos G. 1997. Nutrición y dieta de las poblaciones aborígenes prehispánicas del sudeste de la Región Pampeana. Tesis Doctoral Inédita. Facultad de Ciencias Naturales y Museo. Universidad Nacional de La Plata. https://www.academia.edu/309478/ Nutrici\%C3\%B3n y Dieta de las Poblaciones Abor $\% \mathrm{C} 3 \%$ ADgenes Prehisp $\% \mathrm{C} \overline{3} \% \overline{\mathrm{A}} 1$ nicas del Sudeste de la Regi\%C3\%B3n Pampeana

Behrensmeyer AK, Hill AP. 1980. Fossils in the making. Vertebrate taphonomy and paleoecology. Chicago: University of Chicago Press. doi:10.1017/ s0016756800024894

Bell LS. 1990. Palaeopathology and diagenesis: A SEM evaluation of structural changes using backscattered electron imaging. J Archaeol Sci 17:85-102. doi:10.1016/0305-4403(90)90016-x

Bell LS, Elkerton A. 2008. Human remains recovered from a sixteenth century mass fatality: unique marine taphonomy in human skeletal material from the medieval warship the Mary Rose. Int J Osteoarch 18:523-535. doi:10.1002/oa.952

Bell LS, Skinner MF, Jones SJ. 1996. The speed of post mortem change to the human skeleton and its taphonomic significance. Forensic Sci Int 82:129-140. doi:10.1016/0379-0738(96)01984-6

Borrero LA. 2011. La función transdisciplinaria de la arqueozoología en el siglo XXI: Restos animales y más allá. Antipod Rev Antropol Arqueol 13:267-274. doi:10.7440/antipoda13.2011.13

Breen AP, Murphy JA. 1995. Reactions of oxyl radicals with DNA. Free Radical Bio Med 18:1033-1077. doi:10.1016/0891-5849(94)00209-3

Child AM. 1995. Microbial taphonomy of archaeological bone. Studies in Conservation 40:19-30. doi: $10.2307 / 1506608$

Collins MJ, Galley P. 1999. Towards an optimal method of archaeological collagen extraction: the influence of $\mathrm{pH}$ and grinding. Ancient Biomolecules 2:209-222.

Collins MJ, Nielsen-Marsh C, Hiller J, Smith CI, Roberts JP, Prigodich RV, Wess TJ, Csapò J, Millard AR, TurnerWalker G. 2002. The survival of organic matter in bone: A review. Archaeometry 44:383-394. doi:10.1111/14754754.t01-1-00071

Davidson MW, Abramowitz M. 2002. Optical microscopy. Encyclopedia of imaging science and technology. New York: Wiley-Interscience. doi:10.1002/0471443395. img074

Duday H. 2009. The archaeology of the dead: Lectures in archaeothanatology. Oxford: Oxford University Press. doi:10.1017/s095977431000065x

Efremov I. 1940. Taphonomy: new branch of paleontology. Pan-American Geologist 74:81-93.

Egerton R. 2005. Physical principles of electron microscopy. An introduction to TEM, SEM, and TEM. Berlín: Springer. doi:10.1007/b136495

Fernández-Jalvo Y, Andrews P, Pesquero MD, Smith C, Marín-Monfort D, Sánchez B, Geigl EM, Alonso A. 2010. Early bone diagenesis in temperate environments. Part I: Surface features and histology. Palaeogeogr $\mathrm{Pa}-$ laeoclimatol Palaeoecol 288:62-81. doi:10.1016/j.palaeo.2009.12.016

Galligani PE. 2013. Tafonomía de los entierros humanos del sitio Río Salado-Coronda II. Tesis de Licenciatura Inédita. Facultad de Humanidades y Artes. Universidad Nacional de Rosario.

Garland AN. 1989. Microscopical analysis of fossil bone. Appl Geochem 4:215-29. doi:10.1016/08832927(89)90021-8

Gifford DP. 1981. Taphonomy and paleoecology: a critical review of archaeology's sister disciplines. Advances in Archaeological Method and Theory 4:365-438.

Gilbert TPM, Rudbeck L, Willerslev E, Hansen AJ, Smith C, Penkman KEH, Prangenberg K, Nielsen-Marsh CM, Jans ME, Arthur P, Lynnerup N, Turner-Walker G, Biddle M, Kjølbye-Biddle B, Collins MJ. 2005. Biochemical and physical correlates of DNA contamination in archaeological human bones and teeth excavated at Matera, Italy. J Archaeol Sci 32:785-93. doi:10.1016/j. jas.2004.12.008

Goldstein J, Newbury DE, Joy DC, Lyman CE, Echlin P, Lifshin E, Sawyer LC, Michael JR. 2003. Scanning electron microscopy and $\mathrm{x}$-ray microanalysis. Nueva York: Kluwer Academic/Plenum Publishers.

Gómez Varga JD. 2004. Microscopía electrónica de barrido ambiental. Revista de Plásticos Modernos, Ciencia y Tecnología de Polímeros 571:51-61.

Goodhew PJ, Humphreys J, Beanland R. 2001. Electron microscopy and analysis. Londres: Taylor \& Francis.

Grupe G. 1995. Preservation of collagen in bone from dry, sandy soil. J Archaeol Sci 22:193-199. doi:10.1006/ jasc.1995.0021

Gutiérrez MA. 2001. Bone diagenesis and taphonomic history of the Paso Otero 1 bone bed, Pampas of Argentina. J Archaeol Sci 28:1277-1290. doi:10.1006/ jasc. 2000.0648

Hackett CJ. 1981. Microscopical focal destruction (tunnels) in exhumed human bones. Med Sci Law 21:243-265.

Haglund W, Sorg M. 1996. Forensic taphonomy: the post- 
mortem fate of human remains. Florida: CRC Press. doi:10.1201/9781439821923

Hanson DB, Buikstra JE. 1987. Histomorphological alteration in buried human bone from the Lower Illinois Valley: implications for palaeodietary research. J Archaeol Sci 14:549-563. doi:10.1016/0305-4403(87)90038-0

Hedges REM. 2002. Bone diagenesis: An overview of processes. Archaeometry 44:319-328. doi:10.1111/14754754.00064

Hedges REM, Millard AR. 1995. Bones and groundwater: towards the modelling of diagenetic processes. J Archaeol Sci 22:155-164. doi:10.1006/jasc.1995.0017

Hedges REM, Millard AR, Pike AWG. 1995. Measurements and relationships of diagenetic alteration of bone from three archaeological sites. J Archaeol Sci 22:201-209. doi:10.1006/jasc. 1995.0022

Hollund HI, Ariese F, Fernandes R, Jans MME, Kars H. 2012a. Testing an alternative high-throughput tool for investigating bone diagenesis: FTIR in attenuated total reflection (ATR) mode. Archaeometry 55:507-532. doi:10.1111/j.1475-4754.2012.00695.x

Hollund HI, Jans MME, Collins MJ, Kars H, Joosten I, Kars SM. 2012b. What happened here? Bone histology as a tool in decoding the postmortem histories of archaeological bone from Castricum, The Netherlands. Int J Osteoarch 22:537-548. doi:10.1002/oa.1273

Hollund HI, Jans MME, Kars H. 2014. How are teeth better than bone? An investigation of dental tissue diagenesis and state of preservation at a histological scale (with photo catalogue). Internet Archaeology 36. doi:10.11141/ia.36.7

Hu YW, He DL, Dong Y, Wang CS, Gao MK, Lan YF. 2006. Linear scanning analysis of prehistoric human bones in Xigongqiao site, Tengzhou, Shandong province by use of SEM-EDS. Spectroscopy and Spectral Analysis 26:1179-1182.

Jackes M, Sherburne R, Lubell D, Barker C, Wayman M. 2001. Destruction of microstructure in archaeological bone: a case study from Portugal. Int J Osteoarch 11:415-432. doi:10.1002/oa.583

Jans, MC. 2008. Microbial bioerosion of bone- A review. En: Wisshak M, Tapanila, L, editores. Current developments in bioerosion. Berlin y Heidelberg: Springer. $\mathrm{p}$ 397-413. doi:10.1007/978-3-540-77598-0_20

Jans MC, Kars H, Nielsen-Marsh C, Smith C, Nord A, Earl N. 2002. In situ preservation of archaeological bone: a histological study within a multidisciplinary approach. Archaeometry 44:343-352. doi:10.1111/1475-4754.t01-1-00067

Jans MC, Nielsen-Marsh C, Smith C, Collins M, Kars H. 2004. Characterisation of microbial attack on archaeological bone. J Archaeol Sci 31:87-95. doi:10.1016/j. jas.2003.07.007

King CE, Debruyne R, Kuch M, Schwarz C, Poinar HN. 2009. A quantitative approach to detect and overcome PCR inhibition in ancient DNA extracts. BioTechniques 47:941-949. doi:10.2144/000113244

Lyman RL. 1994. Vertebrate taphonomy. Cambridge: Cambridge University Press. doi:10.1017/ cbo9781139878302

Lyman RL. 2010. What taphonomy is, what it isn't, and why taphonomists should care about the difference. Journal of Taphonomy 8:1-16.

Marchiafava V, Bonuci L, Ascenzi A. 1974. Fungal osteoclasia: a model of dead bone resorption. Calc Tiss Res 14:195-210. doi:10.1007/bf02060295

Mehta R. 2012. Interactions, imaging and spectra in SEM. En: Kazmiruk V, editor. Scanning electron microscopy. Rijeka: InTech. p. 17-30.
Mertz J. 2009. Introduction to optical microscopy. Greenwood Village: Roberts \& Co Publ.

Morales N, Catella L, Barrientos G, Oliva F. 2014. Evaluación de un modelo predictivo acerca de las probabilidades diferenciales de preservación ósea en diferentes sectores de la cuenca del arroyo Chasicó, Región Pampeana Argentina. Actas $12^{\text {th }}$ International Conference of Archaeozoology. San Rafael: Mendoza.

Murphy DB, Davidson MW. 2013. Fundamentals of light microscopy and electronic imaging. Hoboken: WileyBlackwell. doi:10.1002/9781118382905

Nash DT, Petraglia MD. 1987. Natural formation processes and the archaeological record. BAR International Series 352. Oxford: Archaeopress. doi:10.1002/ gea.3340040212

Nielsen-Marsh C, Hedges R. 2000. Patterns of diagenesis in bone I: the effects of site environments. J Archaeol Sci 27:1139-1150. doi:10.1006/jasc. 1999.0537

Ortiz López A. 2011. Los procesos tafonómicos en la formación de los depósitos funerarios. Estrat Crític Revista d'Arqueologia 5:452-460.

Pesquero MD, Ascaso C, Fernández-Jalvo Y, Alcalá L. 2010. A new taphonomic bioerosion in a Miocene lakeshore environment. Palaeogeogr Palaeoclimatol Palaeoecol 295:192-198. doi:10.1016/j.palaeo.2010.05.037

Pesquero MD, Fernández-Jalvo Y.2014. Bioapatite to calcite, an unusual transformation seen in fossil bones affected by aquatic bioerosion. Lethaia 47:533-546. doi:10.1111/let.12079

Piepenbrink H. 1986. Two examples of biogenous dead bone decomposition and their consequences for taphonomic interpretation. J Archaeol Sci 13:417-430. doi:10.1016/0305-4403(86)90012-9

Pokines J, Symes SA. 2013. Manual of forensic taphonomy. Boca Raton: CRC Press. doi:10.1201/b15424

Prowse T, Schwarcz HP, Saunders S, Macchiarelli R, Bondioli L. 2004. Isotopic paleodiet studies of skeletons from the Imperial Roman-age cemetery of isola Sacra, Rome, Italy. J Archaeol Sci 31:259-272. doi:10.1016/j. jas.2003.08.008

Rogel MR, Qiu HJ, Ameer GA. 2008. The role of nanocomposites in bone regeneration. J Mater Chem 18:42334241. doi:10.1039/b804692a

Ross MH, Pawlina W. 2007. Histología. Buenos Aires: Editorial Médica Panamericana.

Shin JY. 2011. Modified bone density fractionation method for palaeodietary studies. Bull Korean Chem Soc 32:8388. doi:10.5012/bkcs.2011.32.1.83

Smith CI, Nielsen-Marsh CM, Jans MC, Collins MJ. 2007. Bone diagenesis in the European Holocene I: patterns and mechanisms. J Archaeol Sci 34:1485-1493. doi:10.1016/j.jas.2006.11.006

Stout SD. 1978. Histological structure and its preservation in ancient bone. Curr Anthropol 19:601-604. doi:10.1086/202141

Turner-Walker G. 2008. The chemical and microbial degradation of bones and teeth. En: Pinhasi R, Mays $\mathrm{S}$, editores. Advances in human palaeopathology. Chichester: John Wiley \& Sons Ltd. p. 3-29. doi:10.1002/9780470724187.ch1

Turner-Walker G. 2012. Early bioerosion in skeletal tissues; persistence through deep time. Neues Jahrb Geol Palaeontol Abh 265:165-183.

Turner-Walker G, Jans MC. 2008. Reconstructing taphonomic histories using histological analysis. Palaeogeogr Palaeoclimatol Palaeoecol 266:227-23. doi:10.1016/j. palaeo.2008.03.024

Turner-Walker G, Mays S. 2008. Histological studies on an- 
cient bone. En: Pinhasi R, Mays S, editores. Advances in human palaeopathology. Chichester: John Wiley \& Sons Ltd. p. 121-146. doi:10.1002/9780470724187.ch7

Turner-Walker G, Syversen U. 2002. Quantifying histological changes in archaeological bones using BSESEM image analysis. Archaeometry 44:161-168. doi:10.1111/1475-4754.t01-1-00078

Turner-Walker G, Nielsen-Marsh CM, Syversen U, Kars H, Collins MJ. 2002. Sub-micron spongiform porosity is the major ultra-structural alteration occurring in archaeological bone. Int J Osteoarch 12:407-414. doi:10.1002/oa.642

Tütken T, Vennemann TW. 2011. Fossil bones and teeth: preservation or alteration of biogenic compositions? Palaeogeogr Palaeoclimatol Palaeoecol 310:1-8. doi:10.1016/j.palaeo.2011.06.020
Van Klinken GJ, Hedges REM. 1995. Experiments on collagen-humic interactions: speed of humic uptake, and effects of diverse chemical treatments. J Archaeol Sci 22:263-270. doi:10.1006/jasc. 1995.0028

Von Endt DW, Ortner DJ. 1984. Experimental effects of bone size and temperature on bone diagenesis. J Archaeol Sci 11:247-253. doi:10.1016/0305-4403(84)90005-0

Weiner S. 2010. Microarchaeology: beyond the visible archaeological record. Cambridge: Cambridge University Press.

Weiner S, Bar-Yosef O. 1990. States of preservation of bones from prehistoric sites in the Near-East: a survey. J Archaeol Sci 17:187-196. doi:10.1016/03054403(90)90058-d

White TD, Folkens PA. 2005. The human bone manual. doi:10.1016/b978-0-12-088467-4.50008-9 